Revue de l'Institut français d'histoire en Allemagne

\title{
« Kanonen statt Fleisch ».
}

L'approvisionnement déclinant en viande de l'Allemagne pendant la Seconde Guerre mondiale (1939-1945)

\section{Michel-Pierre Chelini}

\section{(2) OpenEdition}

1 Journals

\section{Édition électronique}

URL : http://journals.openedition.org/ifha/198

DOI : 10.4000/ifha. 198

ISSN : 2198-8943

\section{Éditeur}

IFRA - Institut franco-allemand (sciences historiques et sociales)

\section{Édition imprimée}

Date de publication : 6 février 2011

Pagination : 224-252

ISSN : 2190-0078

\section{Référence électronique}

Michel-Pierre Chelini, « «Kanonen statt Fleisch ». », Revue de l'IFHA [En ligne], 3 | 2011, mis en ligne le 16 février 2012, consulté le 19 avril 2019. URL : http://journals.openedition.org/ifha/198 ; DOI : 10.4000/ifha. 198

Ce document a été généré automatiquement le 19 avril 2019.

(C)IFHA 


\title{
« Kanonen statt Fleisch ».
}

\author{
L'approvisionnement déclinant en viande de l'Allemagne pendant la \\ Seconde Guerre mondiale (1939-1945)
}

Michel-Pierre Chelini

\section{NOTE DE L'ÉDITEUR}

Michel-Pierre Chélini est professeur d'histoire contemporaine à l'université d'Artois (Arras).

\section{Introduction et sources}

Les restrictions alimentaires liées au conflit ont touché le Reich dès la première année de guerre, malgré les victoires et les prélèvements sur les territoires occupés. Toutes les branches agroalimentaires ont été concernées et le marché de la viande, connu pour son rôle moteur dans l'inflation des années $1940^{1}$, n'a pas échappé à cette diminution générale, pouvant illustrer à sa manière le propos propagandiste de Goebbels en 1935-1936, « Kanonen statt Butter »².

Destiné initialement à une communication de colloque ${ }^{3}$, ce papier a bénéficié du soutien de la MHFA (puis IFHA) pour une mission à Berlin à l'été 2009, et repose principalement sur le dépouillement de dossiers du ministère de l'Agriculture et du Ravitaillement du Reich. Il est étayé par l'étude de la documentation imprimée en France et sur le dépouillement à Paris des dossiers du ministère de l'Agriculture français sous Vichy ${ }^{4}$.

Sur le site berlinois du Bundesarchiv, à Berlin Lichterfelde, deux fonds ont été sondés principalement : la série $\mathrm{R} 3601^{5}$ pour le ministère de l'Agriculture et du Ravitaillement du Reich (Reichsministerium für Ernährung und Landwirtschaft, REL ou RMEL), et la série R $17 \mathrm{~V}$ pour la Confédération corporative allemande de l'élevage ${ }^{6}$ (Hauptvereinigung der Deutschen Viehwirtschaft). Issu d'une administration de guerre créée à partir de 1916, le ministère de l'Agriculture du Reich est devenu un service de plein exercice sous la République de Weimar. En avril-mai 1945, une partie des 2500 dossiers du ministère 
déposés à Potsdam a disparu dans les flammes, de même qu'une bonne partie des papiers utilisés au ministère même, dans la Wilhelmstrasse. Toutefois, une évacuation préventive avait été réalisée aux environs de Landsberg an der Warthe ${ }^{7}$, tandis qu'une autre évacuation programmée vers Kustrin n'a jamais été réalisée. La série est donc extrêmement fragmentaire.

D'autres séries n'ont pas pu être examinées ${ }^{8}$ ou n'ont révélé aucune trace de dossiers en relation avec la viande ${ }^{9}$. Outre le défaut d'investigation dans les archives militaires, appréhendées par bribes sous forme de correspondance entre l'Oberkommando des Heeres (OKH), l'état-major général, et le RMEL, mais qui auraient nécessité un séjour à Fribourg, la recherche a été gênée par l'impossibilité de trouver de la documentation directe sur la Confédération corporative de la boucherie allemande (Reichsinnungsverband des Fleischshandwerks), fondée en 1875. Au carrefour de l'industrie, du commerce et de l'artisanat, la boucherie est parfois difficile à identifier dans les $\operatorname{archives}^{10}$ même si la piste des chambres de commerce a été suggérée tardivement ${ }^{11}$.

En renfort de ce corpus archivistique, la documentation imprimée est plus regroupée en France - quelques thèses de doctorat, plusieurs articles, deux ou trois monographies incluant le marché de la viande - qu'en Allemagne, où elle est plus dispersée dans les périodiques professionnels, alors que le thème $\mathrm{y}$ a été très étudié pendant la Première Guerre mondiale.

Le marché de la viande dans le Reich entre 1939 et 1945 offre une situation paradoxale : les quantités diminuent dès le premier mois du conflit, alors que l'Allemagne l'emporte sur tous les fronts et prélève animaux et carcasses dans les territoires occupés. Comment la ration des civils allemands a-t-elle pu diminuer de plus de moitié en six années de conflit? L'Allemagne a-t-elle cherché à compenser cette diminution par des efforts d'innovation dans le transport, le conditionnement et la conservation, ou bien la priorité accordée à la Wehrmacht a-t-elle déséquilibré l'ensemble du circuit, emportant l'approvisionnement militaire dans sa chute?

Nous examinerons successivement la situation du marché de la viande en 1937-1938, la baisse globale des quantités disponibles pendant le conflit et les implications de la priorité accordée au ravitaillement militaire.

\section{L'offre et la demande de viande en Allemagne.}

Le premier consommateur européen en 1937

\section{Près de $50 \mathrm{~kg}$ par an et par habitant}

En 1937, l'Allemagne compte environ 66 millions d'habitants, soit approximativement 22 millions de ménages, sur une superficie de $470000 \mathrm{~km}^{2}$. Elle forme un peu moins d'un sixième $(17,5 \%)$ de la population européenne ${ }^{12}$. La population urbaine allemande, de l'ordre de 45 millions de personnes (67\%), dépasse la population française totale et forme environ $20 \%$ de la population urbaine européenne. L'agriculture elle-même, sans compter l'industrie agroalimentaire, occupe un peu moins de dix millions d'actifs (30\%). Sur un peu plus de cinq millions d'exploitations, plus de la moitié sont susceptibles de livrer un animal au circuit commercial dans l'année. 
En 1937-1938, l'Europe livre environ 12,5 millions de tonnes de viande ${ }^{13}$. Ces statistiques sont en partie approximatives car elles incluent ou non, selon le pays, la viande de cheval, la volaille et la charcuterie; elles sont présentées tantôt en valeur annuelle reconstituée à partir des données de campagne, tantôt en moyenne quinquennale ${ }^{14}$ et estiment difficilement l'autoconsommation rurale.

L'Allemagne, avec 3,3 millions de tonnes de viande abattue, soit $27 \%$ de la consommation européenne, devance sensiblement la France avec 1,8 million de tonnes (14\%), mais l'écart par habitant est moindre: $47 \mathrm{~kg} / \mathrm{an} / \mathrm{habitant}$ contre $44 \mathrm{~kg}$ en France. Cette consommation augmente depuis le XIXe siècle, mais, depuis 1914, la conjoncture induit une progression plus irrégulière, avec des épisodes de recul en période critique : 45,8 kg en $1929,42 \mathrm{~kg}$ en $1933,43,6 \mathrm{~kg}$ en $1936^{15}$. Le tonnage de viande bovine (incluant le veau) augmente de 800000 tonnes en 1911-1913 à 980000 tonnes en 1936-1938, et celle de porc de 1,4 million de tonnes à 2 millions pendant la même période ${ }^{16}$.

Replacée dans le contexte des pays avancés ou gros producteurs ${ }^{17}$, l'Allemagne apparaît dans les consommateurs moyens, comparable aux États-Unis ou à la Grande-Bretagne (60-62 kg/an/hab.), positionnée entre les grands consommateurs comme l'Uruguay, l'Australie ou la Nouvelle-Zélande (110-140 kg/an/hab.) et les consommateurs plus modérés que sont les États méridionaux comme l'Italie $(20 \mathrm{~kg})$. La répartition des types de viandes consommées en Europe montre la prévalence du porc (50\%), devant le bœuf (32\%), loin devant le mouton ( $8 \%$ ) ou le veau (6\%).

Tableau 1. Répartition des types de viandes consommées en Europe, 1938 (en \%) ${ }^{18}$

\begin{tabular}{|l|l|l|l|}
\hline & Europe & Allemagne & France \\
\hline Bœuf & 32 & 33 & 40,4 \\
\hline Porc & 51 & 57,5 & 35,2 \\
\hline Veau & 6 & 6,5 & 17 \\
\hline Mouton & 8 & 1,5 & 5,4 \\
\hline Cheval & {$[3]$} & 1 & 2,7 \\
\hline Total & 100 & 100 & 100 \\
\hline
\end{tabular}

Les Allemands consomment proportionnellement plus de porc (57,5\%) que la moyenne des Européens, autant de bœuf (33\%) et de veau ( $6 \%$ ), mais moins de mouton (1,5\%) et de cheval $(1 \%)^{19}$. La viande de chèvre est résiduelle : $0,3 \%$. La montée du bœuf et le déclin du mouton sont assez caractéristiques de l'évolution européenne depuis le XIXe siècle. Ainsi, le Reich dans ses frontières de 1871 enregistre un doublement de son cheptel chevalin, qui passe de 2,7 millions de têtes en 1850 à 4,5 millions en 1913, tandis que les bovins augmentent seulement de $60 \%$, de 13 millions à 21 millions de têtes ${ }^{20}$. En revanche, la population porcine quintuple, de 5,3 à 25,6 millions, ce qui est une véritable explosion pendant cette période, les ovins suivant le chemin exactement inverse, de 25 millions à 5,5 millions, car la laine vient désormais d'Australie. 
Les disponibilités apparentes en viande varient selon les provinces prussiennes et les länder, en fonction des capacités d'élevage et de la population résidente. Ainsi en 1938, pour une moyenne nationale de $47,1 \mathrm{~kg} / \mathrm{an} / \mathrm{habitant}$, la Bavière très pourvue en cheptel, mais assez peuplée, affiche seulement $45 \mathrm{~kg}$ et le Wurtemberg $42 \mathrm{~kg}$, mais le SchleswigHolstein moins peuplé $78 \mathrm{~kg}$ et le Mecklembourg $64 \mathrm{~kg}$, tandis que la Rhénanie du NordWestphalie, très densément habitée, enregistre $36 \mathrm{~kg}^{21}$. Dans les faits, la consommation se répartit différemment en raison des écarts de niveau de vie et de densité citadine. À côté de ces quartiers plus «nobles », la population consomme également, en quantité difficile à établir, d'autres produits carnés qui augmentent d'un tiers environ l'apport en protéines animales : abats, volaille, lapins, chèvres et gibier ${ }^{22}$.

Cette consommation croise une demande et une offre à un certain prix, dépendant du marché national et international, et des représentations culturelles de la viande dans l'opinion allemande. Il faut savoir que la viande signifie, pour la moyenne de la population de cette époque, force physique et niveau de vie en hausse. La production d'une calorie animale nécessite en effet la consommation préalable de cinq à dix calories végétales par les animaux élevés. Les prix ne sont pas uniquement le marqueur de différences sociales ${ }^{23}$, car les écarts sont d'abord liés à l'âge, au sexe ou aux catégories socioprofessionnelles: les hommes consomment plus que les femmes, les jeunes que les personnes âgées, les ouvriers et les exploitants agricoles que les employés ${ }^{24}$. La qualité des morceaux et le type de viande contribuent aux différences sociales: plus de viande de bœuf dans les ménages aisés, plus de viande de cheval dans les milieux populaires. Les pratiques culturelles et religieuses peuvent influer sur les rythmes de consommation avec l'abstinence du vendredi, la consommation d'agneau à Pâques ${ }^{25}$ ou encore la surconsommation relative de viande les dimanches et jours de fête ${ }^{26}$.

\section{Un marché spécifique}

La viande n'est pas, comme les céréales ou les pommes de terre, le produit d'un cycle fortement annualisé, où la récolte se concentre sur un ou deux mois et apporte le revenu d'une année entière. Elle n'est pas plus le résultat d'un cycle hebdomadaire ou quotidien comme le lait et les œufs, dont la collecte continue approvisionne régulièrement la trésorerie des exploitations. Elle rassemble une pluralité de cycles d'amplitude variable du poulet mensuel à la vache de réforme décennale, en passant par le porc annuel ou le bœuf d'embouche trisannuel ${ }^{27}$. Dans ces conditions, les cycles d'offre et de demande ne se recoupent pas toujours. La demande est assez stable dans l'année, avec une augmentation en saison froide, l'offre s'adapte grâce à l'élasticité de stockage que permettent le cheptel vivant et la présence de circuits frigorifiques pour les carcasses. En 1938, en données trimestrielles, le nombre de porcins sur pied passe ainsi de 20,2 millions en mars (indice 91) à 21,3 en juin (96) puis à 24,2 en septembre (109), avant de reculer à 22,9 millions en décembre $(103)^{28}$. Le pic de juin à octobre correspond à des porcs engraissés grâce aux récoltes de l'année, avant les abattages d'automne-hiver.

Le "producteur de viande » est également un ensemble relativement varié d'exploitants dont les intérêts ne sont pas toujours convergents : petit exploitant qui vend de temps en temps une vache laitière en fin de course ou une paire de bœufs de trait, petit éleveur de porcs, petit ou moyen éleveur qui se consacre exclusivement à la naissance et à l'embouche d'animaux sélectionnés, propriétaire de grandes porcheries industrielles qui 
engraisse des milliers de porcs achetés jeunes dans les régions d'élevage, gros exploitant qui achète chaque année cent à deux cents bovins maigres ou semi-gras à engraisser.

Tableau 2. Cheptel bovin des exploitations de plus de 0,5 ha $(1933)^{29}$

\begin{tabular}{|l|l|l|l|l|}
\hline Taille des exploitations & Nombre d'exploitations & $\begin{array}{l}\text { Cheptel bovin } \\
\text { en millions }\end{array}$ & $\begin{array}{l}\text { \% des } \\
\text { exploitations }\end{array}$ & \% des animaux \\
\hline$<5$ ha & 1249000 & 3,3 & 44 & 15 \\
\hline $5-20$ ha & 1119000 & 8,8 & 39,5 & 40,9 \\
\hline $20-100$ ha & 370000 & 7,15 & 13 & 33 \\
\hline$>100$ ha & 29500 & 2,1 & 1 & 9,7 \\
\hline Total & 2840000 & 21,5 & 100 & 100 \\
\hline
\end{tabular}

La grande majorité des exploitations allemandes élève du bétail, puisque sur un total général de 5,5 millions d'exploitations, près de 2,8 millions de celles qui dépassent le demi-hectare comptent au moins un bovin- chiffre comparable aux 2,3 millions d'exploitations françaises dans ce cas. On constate sans surprise que les petites exploitations de moins de 5 ha forment $44 \%$ du total et rassemblent $15 \%$ des bovins, tandis que les exploitations de plus de 100 ha, moins d' $1 \%$ du total, comptent près de $10 \%$ des bovins. La proportion est équilibrée auprès des $5-20$ ha, $40 \%$ des exploitations et $40 \%$ $\mathrm{du}$ cheptel bovin, tandis que les exploitations cossues de $20-100 \mathrm{ha}, 13 \%$ du total des entreprises agricoles, rassemblent le tiers de tout le cheptel. Les chiffres des porcins sont extrêmement proches, avec 2,9 millions d'exploitations concernées ${ }^{30}$.

La répartition géographique de l'élevage en Allemagne est relativement régulière. Pour les bovins, on note deux zones de plus fortes densités de cheptel : la frange océanique qui relie le Schleswig-Holstein à la Westphalie occidentale, via les Pays-Bas et la frange montagneuse qui court de la Silésie à la Bavière, en bordant la frontière tchèque. Dans tous les länder ou provinces, bovins et porcins sont souvent associés, mais en Bavière, Bade, Wurtemberg et Schleswig, les bovins sont plus nombreux que les porcins. L'est de l'Elbe est moins pourvu en bovins ${ }^{31}$ et le centre de gravité de l'élevage porcin est la Westphalie et la Basse-Saxe occidentale.

L'herbager important ou l'engraisseur industriel, producteurs de viande, ne sont pas des exploitants comme les autres : ils sont aussi acheteurs (et donc commerçants) auprès des « naisseurs » à certaines époques de l'année, avec des intérêts divergents. À l'opposé, le cultivateur qui pratique surtout la polyculture n'est qu'accessoirement un producteur de viande. Quand il vend une bête une fois dans l'année, et de préférence au prix fort, il risque de devoir acheter de «la viande » chez son boucher détaillant, également à prix fort. Le prix élevé de la viande n'est pas nécessairement favorable pour lui. Ainsi, le « circuit de la viande » n'offre pas, dans la partie élevage, la cohésion professionnelle que l'on peut retrouver dans le blé, la betterave ou le lait ${ }^{32}$.

Pour finir, la production animale permet des transferts internes entre les issues de la filière. Le cœur du troupeau est constitué par les vaches : sur 20,5 millions de «bovins » en 1937, les veaux de moins de trois mois constituent un groupe de 1,5 million de têtes, 
les jeunes boufs ou génisses de trois mois à deux ans près de 6,7 millions, les taureaux 750000 unités et les vaches forment la majorité avec 11,4 millions de têtes ${ }^{33}$. Selon les prix offerts, une vache peut fournir de la viande, du beurre, du lait ou des veaux. On ne peut pas garder son blé sur pied une fois qu'il est mûr, alors qu'un bovin peut être conservé encore quelques mois selon l'évolution des cours. En 1937, le Reich produit environ 25 millions de $\mathrm{m}^{3}$ (tonnes) de lait à l'aide de dix millions de vaches laitières, au rendement moyen annuel de 2490 litres par animal. Un dixième de ce lait sert à l'alimentation des veaux, $11 \%$ est consommé frais par les exploitants, $12 \%$ sert à faire du beurre et $65 \%$ est livré, dont près de $60 \%$ à des laiteries manufacturières ${ }^{34}$.

\section{La guerre : une offre de viande qui diminue sur tout le continent}

\section{Malgré les victoires, la baisse des disponibilités en viande dans le Reich}

À partir de 1938 et des premières annexions, la population du Reich évolue à la hausse, même si le statut des territoires annexés n'est pas toujours assimilé à celui des Allemands volksdeutsch. Vers 1941, le IIIe Reich est devenu un État gigantesque de $840000 \mathrm{~km}^{2}$ avec une population officielle de 110 millions d'habitants ${ }^{35}$. Il contrôle de près ou de loin cinq millions de $\mathrm{km}^{2}$ et plus de 300 millions d'habitants en Europe seule. Malgré les victoires et des prélèvements sur les pays occupés, les disponibilités allemandes en viande diminuent dès l'automne 1939. Si la France vaincue subit une réduction des disponibilités en viande de l'ordre de $40 \%$ environ ${ }^{36}$, on peut expliquer la déperdition par les prélèvements de l'occupant ou le marché noir. Ces arguments ne peuvent fonctionner pour le Reich.

Tableau 3. Viande disponible dans le Reich 1938-1944 (sans gras ni abats) ${ }^{37}$

\begin{tabular}{|l|l|l|l|l|}
\hline & $\begin{array}{l}\text { Total } \\
\text { (millions de t. })\end{array}$ & $\begin{array}{l}\text { Porc } \\
\text { (millions de t.) }\end{array}$ & $\begin{array}{l}\text { Bœuf } \\
\text { (millions de t.) }\end{array}$ & $\begin{array}{l}\text { Disponibilités } \\
\text { (kg/habitant) }\end{array}$ \\
\hline 1938 & 3,25 & 1,83 & 1,08 & 47,1 \\
\hline 1939 & 3,36 & 2,07 & 1,01 & 45,5 \\
\hline 1940 & 2,29 & 1,80 & 0,91 & 42,9 \\
\hline 1942 & 2,37 & 1,30 & 0,78 & 33,5 \\
\hline 1943 & 1,86 & 0,93 & 0,69 & 25,9 \\
\hline 1944 & 1,68 & 0,91 & 0,52 & 22,1 \\
\hline
\end{tabular}

La situation est clairement celle d'un effondrement. En 1944, les disponibilités apparentes représentent environ la moitié ( $52 \%$ ) de celles de 1938 - encore moins dans la réalité, en raison des priorités accordés aux soldats et aux travailleurs de force. L'entrée en guerre marque le premier palier de la chute (-32 \%). Après un regain en 1941-1942, le passage à la 
« guerre totale » puis l'accumulation des défaites amorcent une seconde phase de recul. Le porc est particulièrement touché, régressant de $57,5 \%$ du tonnage global de viande abattue à $54 \%$ en 1944 , tout comme le bœuf qui recule de $33 \%$ à $31 \%$. Le veau se maintient mieux (6,5\% en 1938 et $10,6 \%$ en 1944 , mais avec $10 \%$ de tonnage en moins) car son cycle est plus court; la viande de mouton déjà confidentielle diminue en proportion, la viande de cheval remonte, de $1 \%$ à $2 \%$, avec un tonnage resté stable (33 000 à 34000 tonnes).

Ces $52 \%$ de disponibilités en 1944 se répartissent différemment selon les régions. La Bavière, les régions à l'est de l'Oder et de la Neisse (57\% du niveau de 1938) et la future zone française d'occupation (56\%) sont un peu moins touchées que la Rhénanie du NordWestphalie $(50 \%)$ ou la future zone soviétique $(49,5 \%)^{38}$. Le nombre d'animaux livrés diminue, par exemple les bœufs rétrogradent de 4,2 millions de têtes en 1938 à 2,4 millions en 1944, mais ils sont moins lourds (-10\%), $255 \mathrm{~kg}$ pour un bovin moyen en 1938 et $231 \mathrm{~kg}$ en 1944. La baisse des rendements est générale : 2265 litres de lait par vache et par an en 1944 contre $2492(-10 \%)$, même si celle-ci est atténuée par une moindre mobilisation extérieure des actifs féminins $\mathrm{s}^{39}$. Le nombre de porcs abattus rétrograde de moitié, de 22,6 millions en 1938 à 11,8 millions en 1944, mais surtout la part de l'abattage à la ferme grimpe de $38 \%$ du total porcin en 1938 à $41,5 \%$ en 1944 après un pic de $59 \%$ en 1942 ! L'abattage non déclaré augmente certainement, sans que l'on puisse exactement l'évaluer. Toutes ces données offrent un parallélisme frappant avec celles de la France, alors que le Reich est vainqueur et impose des prélèvements à ses vaincus, dont la France.

\section{Organisation de la guerre, désorganisation de l'agriculture}

Avec la guerre, la production est affectée par plusieurs facteurs que les efforts de programmation militaire et administrative ne parviennent pas à compenser. Même au temps des victoires, la main-d'œuvre fait rapidement défaut dans les exploitations: autour de $30 \%$ des effectifs combattants sont des actifs agricoles, soit environ 600000 hommes pour les campagnes 1939-1940, un million et demi pour l'offensive contre l'URSS, près de trois millions en 1944. Les activités sont clairement attribuées dans les exploitations : si les femmes s'occupent de la traite des vaches et de la basse-cour, le gros bétail et les porcs sont plutôt du domaine masculin. Les grandes exploitations à l'est de l'Elbe voient partir une fraction de leurs salariés, les petites et moyennes exploitations à l'ouest de la Weser des fils et des neveux. Le remplacement ${ }^{40}$ par de la main-d'œuvre polonaise, balte, biélorusse ou russe, très généralement féminine, voire par des prisonniers de guerre occidentaux ${ }^{41}$, ne compense pas ces départs.

L'agriculture allemande a déjà atteint un certain niveau technique, que la mobilisation militaire perturbe fortement. Sans dégâts matériels internes avant 1943, le territoire du Reich souffre rapidement de la difficulté des transports, eux-mêmes aux effectifs masculins réduits et aux attributions en charbon très mesurées, et des priorités pour les convois militaires. Les engrais, les aliments de bétail importés des États-Unis, les pièces détachées pour les machines, l'approvisionnement en courant électrique pour les trayeuses, tout devient plus laborieux. Les vétérinaires sont moins nombreux, car une partie est également mobilisée pour l'encadrement sanitaire des animaux qui servent à la Wehrmacht - chevaux de transport, chiens de surveillance, animaux d'abattage.

Le quasi-blocage des prix joue un rôle aggravant, car le régime empêche par tous les moyens les prix officiels de monter et menace des pires sanctions les fauteurs de marché 
noir. Avec une division des disponibilités par deux, les prix auraient dû plus que doubler, alors que les prix de gros agricoles en général n'augmentent que de $14 \%$ entre 1938 et 1944, les prix de gros du bœuf, poids vif à l'abattage, de $30 \%$, mais seulement en 1944, car ils sont restés stables jusqu'en 1943 et les prix de gros de la viande de porc de $34 \%^{42}$. De leur côté, les prix de détail alimentaires augmentent de $13 \%$, ceux de la viande ne bougent pas, le litre de lait progressant de $8,5 \%$ et celui du beurre de laiterie de $14,5 \%{ }^{43}$. Les revenus salariés augmentent pendant la même période de 12 à $14 \%$ environ en valeur nominale, mais avec une hausse des prix pratiquement comparable (12\%), leur hausse réelle est de $1 \%{ }^{44}$.

\section{L'encadrement étroit du circuit commercial de la viande}

À partir de 1933, le ministère de l'Alimentation et de l'Agriculture - fondé sous le titre de Reichsministerium für Ernährung und Landwirtschaft en mars 1920 - est confié en 1933 à Walther Darré, favorable au retour à un État agrarien de type "Blut und Boden», tandis que le secrétariat d'État est placé sous la responsabilité de Herbert Backe, proche des thèses d'expansion économique et industrielle du Plan de quatre ans de Goering. Le second supplante le premier, de fait à partir de 1942, de droit après 1944.

La politique économique générale échappe au seul ministère de l'Agriculture et ses papiers ne reflètent l'orientation gouvernementale que de manière indirecte. Les archives ont conservé par exemple la trace assez complète d'un vif débat entre Goering, Darré et Backe en janvier-février 1941, sur l'abaissement de la ration civile de viande de 500 à 400 $\mathrm{g}$ par semaine au 1er juillet $1941^{45}$. En fait, Backe, qui est très proche de Goering et dispose d'un poste de chargé de mission au ministère du Plan de quatre ans, lui fournit, sans que son ministre Darré accepte de signer le document, un état très précis des réserves nationales disponibles en céréales panifiables et fourragères. Cela permet à Goering d'argumenter en faveur d'une réduction de la ration, en relation avec la préparation secrète de la guerre contre l'URSS - jamais mentionnée dans les dossiers traités. Un échange tendu de correspondances entre Darré et Backe est conservé, dans lequel il apparait que le directeur du ministère Moritz, qui reste en poste jusqu'en 1945, prend partie pour le secrétaire d'État Backe. L'intégration du Reichsnährstand au sein du ministère de l'Agriculture (1942), dont Darré était le Reichsbauernführer, scelle la mise à l'écart de ce dernier, dont les options agrariennes étaient devenues contradictoires avec les objectifs productivistes de la " guerre totale ».

$\mathrm{Au}$ sein de l'administration, les classiques débats d'attribution de compétence apparaissent assez régulièrement, jusqu'à la peau d'intestin de porc qui sert à faire les saucisses, le ministère de l'Agriculture étant chargé de la peau naturelle et le ministère de l'Économie de la peau fabriquée dans les usines ${ }^{46}$. Il fallait y penser...

\section{Les éleveurs}

Le premier stade du circuit est celui de l'élevage. Pendant la guerre, se poursuit très régulièrement le 3 décembre (parfois le 2 ou le 4 , si le 3 est un dimanche) le recensement annuel du bétail ${ }^{47}$, prolongeant l'effort statistique des régimes précédents. Les données conservées, de 1936 à 1944, témoignent à la fois d'un grand souci du détail par espèce et par arrondissement (plus exactement par Viehwirtschaftsverband, Union des éleveurs de bétail, il y en a un peu plus de 200 dans le Reich), mais aussi des problèmes de comptage 
liés à l'extension de l'Allemagne : intégration de l'Alsace et de la Moselle à partir de 1940, décomposition des totaux ensuite entre Reich de 1937, Reich de la fin de l'année 1938, Grand Reich avec territoires polonais occupés, etc.

Conscientes de la dégradation de l'offre intérieure et de la contribution des exploitants à cette restriction, les autorités agricoles s'efforcent d'encadrer les «abattages domestiques $»^{48}$ qui désignent tout abattage hors d'un abattoir « industriel » (gewerbliche Schlachtungen). Trois catégories de bénéficiaires distinguent les familles d'exploitants agricoles incluant les salariés agricoles et forestiers (catégorie A), les ruraux non agricoles comme les propriétaires terriens, les artisans, les instituteurs, les médecins ou les curés de campagne (B) et les collectivités comme les hôpitaux ou les établissements d'enseignement (C). Les ouvriers des mines et les ouvriers forestiers sont admis au bénéfice de l'abattage domestique, mais pas les autres salariés du même secteur. Le nombre de ces abattages domestiques ne cesse de croître jusqu'en 1942-1943, avant de se tasser officiellement ensuite. En contrepartie, les bénéficiaires de cet abattage doivent tenir une stricte comptabilité de leurs quantités sur un livret spécial, se délivrer à euxmêmes des coupons, dont des échantillons modèles ont été conservés, respecter les rations par adulte et par enfant de moins de six ans, respectivement $40 \mathrm{~kg}$ et $20 \mathrm{~kg}$ nets par an dans les groupes A et B. Des tables de conversion entre poids vif et poids net (après abattage) sont établies pour trois ensembles géographiques différents ${ }^{49}$. Le ministère est sans illusion sur les dépassements réels, qui excèdent les possibilités de contrôle administratif. Les exploitants peuvent étendre sans mal le calcul des rations aux salariés déportés ou aux prisonniers qu'ils emploient, comme aux sinistrés qu'ils sont contraints d'accueillir en nombre grandissant après 1943.

Le marché du bétail est organisé de manière classique, mais l'encadrement semble augmenter avec la mobilisation militaire, sans que l'on sache vraiment s'il s'agit d'une meilleure collecte animale ou d'une meilleure appréhension statistique. Près de 210 commissions d'achat et de classification des confédérations d'élevage ${ }^{50}$ se mettent sur pied : on en compte par exemple treize en Bade et trente-cinq en Wurtemberg, et le système s'étend aux territoires " assimilés » comme la Bohême-Moravie. La commission moyenne est formée de cinq à dix membres, avec des pointes dans les grands marchés comme Munich ( 22 membres). Chaque commission supervise les ventes de bétail dans les 47 grands marchés allemands (par exemple Mannheim) et les marchés intermédiaires (par exemple Heidelberg). Des centres de pesage (Wiegestellen) sont installés, parfois depuis longtemps, au plus près des exploitants - ainsi l'administration en recense 650 en Bohême-Moravie ${ }^{51}$. En revanche, les dossiers conservés du RMEL ne semblent pas avoir d'informations spécifiques sur les marchands de bétail, qui sont environ 15000 à 20000 en France à la même époque.

Le chiffre total des abattoirs n'a pas été trouvé (on en compte 1500 en France, de toute taille et de tout niveau technique) mais l'administration allemande a recensé les principaux centres d'abattage qui disposent d'une connexion ferroviaire. Autriche incluse, il y en a 157 en 1938-1939 et 174 en $1943^{52}$, en y associant les provinces polonaises perdues par l'Allemagne en 1919. La liste donne une idée de la capacité hebdomadaire d'abattage en équivalent bovins : 500 têtes en moyenne par abattoir ${ }^{53}$, avec de grands abattoirs très modernes comme Berlin (7 000 têtes/semaine), Breslau ou Hambourg (4 000 ) et de petits abattoirs ruraux comme Aschersleben dans l'arrondissement de Magdebourg (40) ou Hattingen dans l'arrondissement d'Arnsberg (20). 
Les statistiques hebdomadaires d'abattage sont très détaillées ${ }^{54}$, par semaine, par types d'animaux en comparaison avec la même semaine, l'année précédente, etc., mais sont toujours établies dans cette série par tête de bétail vif et non par tonnage de viande disponible. Le recensement de 1943 donne la provenance géographique des animaux et montre qu'à ce moment du conflit, l'Allemagne ne cesse d'importer du bétail: en moyenne près de deux animaux abattus sur trois viennent de l'extérieur du Reich.

On ne trouve que peu d'indications sur les bouchers en gros (il y en a 5000 en France), qui appartiennent aux chambres de commerce - artisanat ou industrie - et ne relèvent donc pas de l'agriculture. Ne figurent dans les dossiers étudiés que la réglementation juridique et sanitaire de l'abattage en gros ${ }^{55}$ et la situation des bouchers grossistes de Prague ${ }^{56}$.

\section{Les bouchers de détail}

Une fois les animaux abattus, ils sont mis à la disposition des bouchers de détail sous la forme de quartiers et de morceaux. La commercialisation en gros de viandes s'effectue dans 47 marchés à caractère national (Märkte) 24 marchés intermédiaires (Mittelmärkte) qui leur servent de relais et 215 bureaux de répartition (Verteilungsstellen) qui semblent correspondre aux 200 commissions de vente du bétail citées plus haut ${ }^{57}$. Ces derniers, qui approvisionnent des zones de 300000 habitants environ, sont réglés par un registre hebdomadaire sur lequel les bouchers détaillants déclarent la viande achetée et vendue aux particuliers. Ainsi en Bade, l'administration définit en 1937 neuf Verteilungsstellen en fonction du nombre d'habitants à desservir : Baden-Baden (70 000 hab.), Offenburg (20 000), Lahr (20 000), Lörrach (20 000), Bruchsal (33 000), Constance (40 000), Singen (20 000), Radolfzell (8 000) et Villingen (14 000).

Les bouchers de détail n'apparaissent qu'indirectement dans les sources du RMEL ${ }^{58}$, qui laissent entendre le chiffre d'environ 100000 entreprises artisanales et industrielles de boucherie, depuis les boutiques de quartier jusqu'aux établissements manufacturiers. En France, à pareille époque, il y a entre 40000 et 50000 bouchers pour 40 millions d'habitants, soit un boucher pour 900 habitants, mais la France est rurale à près de $50 \%$. Si l'on applique un coefficient simple à la population allemande, il faut envisager au moins 70000 bouchers de détail pour l'Allemagne dans ses frontières de 1937. Pour Berlin, on compte dans les années 1930-1940 environ 5000 boucheries de détail, soit un boucher pour 800 habitants. Par ailleurs, l'industrie mécanique et électrique allemande livre environ 3000 outils et instruments de travail à l'activité bouchère. Les bouchers sont organisés en profession fédérale depuis 1875 dans le Deutscher Fleischerverband, transformé depuis 1933 en Reichsinnungsverband des Fleischshandwerks ${ }^{59}$.

\section{Une priorité de plus en plus pesante, nourrir la Wehrmacht}

\section{Le rationnement des civils allemands : de $900 \mathrm{~g}$ hebdomadaires (1939) à $300 \mathrm{~g}$ (1942)}

Les dossiers consultés évoquent nettement moins la consommation civile qui est la première touchée par la contraction des disponibilités. Les réquisitions dans les territoires occupés ne suffisent pas à compenser la chute de la production nationale et 
vont surtout nourrir les soldats de la Wehrmacht : de 270000 tonnes en 1940-1941 à 625 000 tonnes en 1942-1943, avant de reculer à 450000 tonnes en 1943-1944 ${ }^{60}$.

Les autorités qui consignent par écrit tous les tonnages abattus et vendus, semaine par semaine, connaissent bien cette situation de pénurie. Depuis septembre 1939, les consommateurs sont divisés en catégories dont le nombre finit par atteindre seize en 1945. Chaque consommateur reçoit des tickets de rationnement et doit acheter la viande à laquelle il a droit dans la boucherie la plus proche de son domicile; même au restaurant, il faut des tickets de rationnement. Les niveaux de viande autorisés sont révisés chaque mois.

La consommation réelle de 1938 était environ de $950 \mathrm{~g}$ par semaine, ce qui équivaut à un peu plus de $120 \mathrm{~g}$ par jour. Chaque offensive aboutit à une réquisition massive de viande pour les troupes et réduit la ration des civils. Dès septembre 1939, la ration civile diminue à $700 \mathrm{~g}$, puis en avril 1940 à $500 \mathrm{~g}$, en juillet 1941 à $400 \mathrm{~g}$, et après février 1942 et la décision de " guerre totale » à $300 \mathrm{~g}$. Les rations militaires parviennent à demeurer assez hautes, leur total passant de $10 \%$ de la consommation globale en septembre 1939 (deux millions de soldats) à $30 \%$ en 1944 (dix millions de soldats) ${ }^{61}$. Malgré la mobilisation d'au moins huit à neuf millions d'Allemands (Reich de 1937) sous l'uniforme, demeurent environ 58 millions de civils qu'il faut ravitailler, auxquels s'ajoute une population de plusieurs millions de personnes déportées ou emprisonnées. Théoriquement bénéficiaires des mêmes rations déclinantes que les Allemands de même statut - les ouvriers ( Zwangsarbeiter) doivent avoir des rations proches de celles des ouvriers allemands - ces populations sont moins nourries en moyenne et surtout connaissent des situations d'une variété assez importante (camps d'officiers prisonniers, de soldats, travailleurs forcés, prisonniers des camps de concentration, des camps d'extermination).

\section{Les rations de la Wehrmacht : de 1,7 kg hebdomadaires (1939) à $600 \mathrm{~g}(1942)$}

Le chiffre total des soldats n'étant jamais donné explicitement et variant dans l'année, les calculs moyens par individu sont très approximatifs. Le dossier a été bien étudié pour l'ensemble du ravitaillement militaire sous plusieurs angles : son organisation, les rations caloriques et vitaminiques, la surveillance sanitaire et médicale ${ }^{62}$. La Wehrmacht consomme pendant les six années de guerre entre 800000 et 600000 tonnes par an, soit 70 à $50 \mathrm{~kg}$ par soldat, ce qui représente des rations plus élevées que celles des civils jusqu'en 1942, puis de moins en moins importantes à partir de 1943-194463. Un tableau récapitulatif établi a posteriori donne des rations de $220 \mathrm{~g}$ à $300 \mathrm{~g}$ par jour et par soldat en campagne entre septembre 1939 et juin 1941 (90 kg/an), mais $200 \mathrm{~g}$ à partir de janvier $1942(70 \mathrm{~kg} / \mathrm{an})$ et un peu moins de $100 \mathrm{~g}$ en $1944(32 \mathrm{~kg} / \mathrm{an})^{64}$. L'armée stationnée à l'intérieur du Reich a toujours des rations inférieures de $25 \%$, jusqu'à l'estompage de la distinction Heimatsgebiet/ Feldgebiet en 1944.

Les dossiers fonctionnent par «année de campagne militaire » depuis le lancement du conflit, en septembre 1939. Pendant la période qui va du 1er septembre 1942 au 31 août 1943, soit le quatrième Kriegswirtschaftsjahr, la Wehrmacht a consommé 753000 tonnes de viande, soit environ $75 \mathrm{~kg} / \mathrm{an} / \mathrm{homme}$ ou $200 \mathrm{~g}$ par jour. L'année 1943-1944 est moins fournie : 653000 tonnes, soit $65 \mathrm{~kg} / \mathrm{an} / \mathrm{homme}$ ou $180 \mathrm{~g}$ par jour ${ }^{65}$. S'il semble que la SS ne soit pas incluse dans ces chiffres, que dire des soldats "étrangers " sous l'uniforme allemand et des auxiliaires volontaires (Hilfswillige)? La Kriegsmarine apparaît 
ponctuellement dans les dossiers ${ }^{66}$, mais pas la Luftwaffe que Goering cherche à conserver dans son orbite ; elle élève par exemple des moutons sur les aérodromes ${ }^{67}$.

Les dossiers comptent beaucoup d'états détaillés des «besoins" de l'OKH, des consommations réelles, des prélèvements sur tel ou tel pays. Un comptage de mars 1943 donne une consommation de $28 \%$ dans les territoires soviétiques occupés, de $21 \%$ dans les territoires considérés comme allemands - Heimatskrieg -, de $16 \%$ dans les autres territoires occupés comme la France et de $34 \%$ à la disposition de la Reichsstelle für Tiere und tierische Erzeugnisse, l'office du Reich pour les Produits animaux, qui répartit entre les réserves, les autres institutions et peut-être certains civils allemands ${ }^{68}$.

Les consommations alimentaires militaires supposent des conditions particulières de stockage, de transport et même de cuisine, surtout en théâtre d'opérations. Certains dossiers donnent pour les consommations en saucisses, jambon, conserves, des valeurs statistiques très détaillées par types de viande. Trois particularités de la viande «militaire» méritent d'être mentionnées (valeurs pour 1943): $30 \%$ de la viande est disponible en conserves, $25 \%$ sous forme de viande congelée et $20 \%$ sous forme de bétail vivant, abattu directement par l'intendance militaire. Les $25 \%$ restants sont vraisemblablement issus de fournitures directes par les circuits bouchers, les exploitants ou les territoires occupés, comme dans le cas de ce boucher de Wünsdorf en Brandebourg qui approvisionne en direct l'école de blindés et l'hôpital situés sur le territoire de la commune ${ }^{69}$. Des tableaux d'équivalence (Anrechnungssätze) sont établis pour comptabiliser ce qui est livré à l'armée, en moyenne $100 \mathrm{~kg}$ sans os représentant $125 \mathrm{~kg}$ à 130 kg livrés. Des tractations apparaissent quand les temps sont durs : le ministère de l'Agriculture propose sans grand succès le remplacement partiel du déficit en viande par de la poudre d'œuf (Trockeneipulver) ${ }^{70}$, du fromage, etc.

On peut considérer que le volume des conserves atteint jusqu'à $30 \%$ du total ${ }^{71}$. Le tonnage net fait l'objet d'une conversion classique de 1,25 en raison de la présence des os : les 150 000 tonnes «valent» ainsi 177000 tonnes dans l'estimation des «Besoins de la Wehrmacht pour 1944-1945 ». La " viande » en conserve elle-même ne représente qu'un peu plus de la moitié du total ; un tiers provient par exemple de conserves de saucisses ${ }^{72}$. Cette consommation pose en permanence le problème des boîtes de conserve, dont le volume est énorme : 176 millions de boîtes de $850 \mathrm{~g}$ et 20 millions de boîtes de $200 \mathrm{~g}$ pour l'année 1944-1945 par exemple ${ }^{73}$. Une active politique de recyclage, de fabrication et de substituts cherche à compenser le risque permanent de rupture de stock ${ }^{74}$. Les efforts d'innovation technique conservés dans les dossiers apparaissent modestes, malgré les tentatives de mise au point de boîtes en aluminium ${ }^{75}$. Il est possible que la documentation du ministère de l'Industrie, des chambres de commerce, des instituts de recherche ou des entreprises industrielles donne plus d'information.

Une autre source évoque également 25 \% de viande congelée ${ }^{76}$, ce qui suppose une chaîne du froid relativement organisée. Les améliorations dans la réfrigération ou l'extension des capacités sont souvent stoppées, faute de matériaux de construction ${ }^{77}$. D'autres innovations ont été signalées par des collègues, notamment pour les produits déshydratés 78 .

Enfin, la Wehrmacht dispose d'unités d'abattage (Schlächtereinheiten) dans ses services d'intendance et de cuisine, qui abattent par exemple 100000 bovins et 25000 porcins par mois en $1944^{79}$, avec un total de 10000 tonnes de viande et cinq millions de saucisses produites mensuellement. Notons la forte prépondérance des bovins, atypique dans le régime alimentaire allemand d'avant-guerre. L'office statistique du Reich leur demande 
d'ailleurs cette année-là de préciser leurs statistiques car avec le repli militaire, leur « production » se fait de plus en plus dans le Reich et devrait rejoindre celle de l'abattage intérieur, ce à quoi l'OKH fait savoir qu'il ne souhaite pas divulguer les chiffres pour des raisons stratégiques ${ }^{80}$.

Les livraisons par les pays occupés sont mentionnées, mais les informations sont meilleures pour les territoires occidentaux comme la France, le Danemark ou la Norvège. $\mathrm{Au}$ total, la France subit un prélèvement moyen de 200000 tonnes par an ${ }^{81}$ qui contraint les éleveurs à fournir des animaux de plus en plus jeunes et moins lourds. Ainsi pendant la quatrième année de guerre, septembre 1942-août 1943, on compte 253000 tonnes livrées par la France. En août 1943, la répartition des 25000 tonnes mensuelles prélevées est la suivante : $24 \%$ pour les troupes présentes en France, $7 \%$ pour celles de la France du Nord (zone interdite, Nord-Pas-de-Calais), 3,5\% pour l'Alsace et la Lorraine, 14,5\% pour les conserves, $8 \%$ pour la viande congelée et $39 \%$ pour le Reich (plutôt pour la Wehrmacht en Heimatkriegsgebiet) ${ }^{82}$. Pour la France, la documentation est assez abondante, preuve de fournitures à la fois importantes et sévèrement marchandées. Dans chaque pays occupé correspondent les dossiers nationaux équivalents ${ }^{83}$. En France, le secrétariat d'État à l'Agriculture a constitué, à l'intérieur de son service des relations extérieures, un service des relations avec les autorités allemandes. Son interlocuteur est la Wirtschaftsabteilung III «Alimentation et agriculture ", dirigée par le Dr Reinhardt. Parfois, l'entretien est important ; le 22 août 1942, on note la présence de deux ministres, Le Roy Ladurie et Bonnafous, mais il s'agit généralement de rencontres de routine une fois par semaine avec quatre interlocuteurs, deux Allemands et deux Français. Les questions concernant la viande y semblent peu abordées.

Le Danemark est très largement prélevé. Le ministère et la Wehrmacht s'étonnent même que la viande y soit vendue sans ticket dans les restaurants et les cantines, ce qui doit être une exception dans toute l'Europe. Cette abondance conduit à un prélèvement de 180000 tonnes pour la cinquième année de guerre ${ }^{84}$. La Norvège, moins pourvue en bovins, n'est imposée qu'à hauteur de 6000 tonnes $^{85}$. L'Italie, alliée, mais occupée après l'armistice du 3 septembre 1943, fait également l'objet de réquisitions sur les marchés officiels pour la Wehrmacht et l'expédition de viande en Allemagne ${ }^{86}$.

Avec le repli de l'été 1944, les soldats et leurs officiers organisent «l'évacuation » des biens "menacés», notamment du bétail ${ }^{87}$ et de la viande considérés comme " Räumungsgut ", " bien évacué ». Le rapatriement prend une telle ampleur que le ministère de l'Agriculture rappelle énergiquement que la limite par soldat est fixée à $75 \mathrm{~kg}$, ce qui représente tout de même la ration civile annuelle de cinq personnes ${ }^{88}$.

\section{Conclusion}

Le marché de la viande dans le Reich entre 1939 et 1945 n'échappe pas au paradoxe du ravitaillement allemand pendant la guerre. Les quantités disponibles diminuent dès le premier mois du conflit malgré victoires et exploitation des vaincus. La priorité d'importantes rations ménagées aux soldats, la mobilisation des actifs agricoles, la diminution des fournitures au secteur agricole font très sensiblement baisser l'offre de viande, que les prélèvements sur les territoires occupés ne compensent que modérément pour un tiers environ. L'offre globale étant réduite de moitié en cinq ans, les civils voient leurs rations divisées par trois, tandis que les rations des soldats, initialement deux fois et demie supérieures à celles des civils, subissent un reflux parallèle après 1942. 
L'évacuation de l'Europe occupée s'accompagne de celle d'une partie de son bétail en 1944.

Le ravitaillement de la Wehrmacht présente quelques originalités : $30 \%$ de conserves, $25 \%$ de viande congelée, $20 \%$ d'animaux abattus directement par l'intendance. Luftwaffe et SS disposent de leurs propres réseaux d'approvisionnement. La priorité accordée à l'équipement militaire se retrouve bien dans l'approvisionnement alimentaire. Les civils allemands en font rapidement les frais, même si la complexité des statuts intermédiaires (auxiliaires, volontaires, civils réquisitionnés), la présence de cantines d'entreprises, l'autoconsommation rurale tempèrent un phénomène extrêmement différencié dans le détail, que les archives fédérales récupérées ne permettent pas d'appréhender avec finesse. Le passage à la guerre totale en 1942 réduit les rations de tout le monde, jusqu'à la crise finale dès l'automne 1944.

La fin de la guerre entraîne celle de l'effondrement de tout le ravitaillement en 1945 dans les zones d'occupation où la situation des quantités et des prix devient très chaotique jusqu'en $1947^{89}$. Les annuaires statistiques respectifs de la RFA et de la RDA ne reprennent qu'en 1952 et 1955, les données qu'ils fournissent débutent généralement en 1948, à l'exception de prix occidentaux disponibles dès juillet 1946. Le premier recensement du bétail à l'Ouest n'a lieu que le 3 décembre 1949, avec la naissance de la RFA ${ }^{90}$. Le marché de la viande en Europe ne se reconstitue pas immédiatement et il faut attendre 1948-1949 environ pour retrouver des conditions stabilisées d'offre et de demande en Allemagne occidentale comme en France.

Au-delà de 1950, la consommation de viande augmente jusqu'aux années 1980. Depuis les années 1990, pour la RFA réunifiée, avec une superficie moindre que celle de 1937 (environ $76 \%$ ), et une population supérieure $(24 \%)^{91}$, le tonnage de viande consommée qui culminait autour de $64 \mathrm{~kg} / \mathrm{an} / \mathrm{habitant}$, a rétrogradé jusqu'à $58 \mathrm{~kg}$ en 2007 . Les épidémies diverses (peste porcine, pic en 1994 en RFA, encéphalite spongiforme bovine en 2000, grippe aviaire en 2006), l'évolution des goûts de consommation en relation avec la santé alimentaire et la prise de conscience écologique ainsi que les réformes de la politique agricole commune, peuvent rendre compte de ces mutations. Le cheptel bovin diminue depuis cette période, de 14,8 millions de têtes en 1999 à 12,7 millions en 2007, tandis que les porcins tendent légèrement à augmenter : 26 millions en 1999, 27 millions en 2007. La viande de bœuf représente environ 1,1 million de tonnes par an en 2007, tout comme la volaille abattue et la viande de porc autour de 5 millions de tonnes.

Les exploitations qui élèvent des animaux (2,8 à 2,9 millions en 1938) sont désormais vingt fois moins nombreuses : autour de 135000 environ, sur un total de 350000 exploitations recensées dont 160000 à temps plein (2005). La concentration est de mise : 39000 exploitations élèvent plus de cent bovins et rassemblent $61 \%$ du cheptel (huit millions sur treize), 90000 élèvent entre vingt et cent bovins pour $61 \%$ du cheptel, quelques milliers élèvent moins de vingt bovins dans des conditions écologiques référencées pour $4 \% \mathrm{du}$ cheptel. Le secteur boucher s'est lui-même concentré, passant de 100000 entreprises en 1938 à environ 15000 actuellement.

\section{Principaux dossiers d'archives dépouillés}

R 3601 Reichsministerium für Ernährung und Landwirtschaft (Mikrofiches)

. R 3601/3153, Fleischversorgung der Wehrmacht 1943-1944 
. R 3601/3154, Fleischversorgung des Protektorates Böhmen und Mähren

. R 3601/3154a, Därme

. R 3601/3154b, Fleischkonserven - Herstellung, Verteilung, Preisbildung 1939-1944

. R 3601/3154c, Einsalzen von Schweinefleisch 1943-1944

.R 3601/3154d, Fleischbewirtschaftung

. R 3601/3162, Selbstversorgung mit Fleisch und Fett 1942-1944 (I)

. R 3601/3179, Selbstversorgung mit Fleisch und Fett (II) Hausschlachtungen.

. R 3601/3189, Bau von Tiefkühlanlagen (Fleisch, Gemüse, Fisch usw.)

. R 3601/3371, Versorgungslage betreffend Getreide und Fleisch 1941

R 17 V Hauptvereinigung der deutschen Viehwirtschaft (Papierakten)

. R 17 V 14, Fleischbilanzen 1942-1945

. R 17 V 27, Märkte, Mittelmärkte, Verteilungsstellen

. R 17 V 29, Viehzählung, 1936-1943

. R 17 V 115, Schlachtviehmarktordnung 1943

\section{Sources administratives imprimées}

Anordnung der Hauptvereinigung der deutschen Viehwirtschaft 1933-1945.

Deutsche Arbeitsfront, Lebensmittelrationen seit dem 25. September 1939 und Einkommen, Berlin : DAF, 1939, 19 p.

Kurt Emig, Das Reichsministerium für Ernährung und Landwirtschaft, Berlin: Junker \& Dünnhaupt, 1939.

Internationaler Verband der Landwirtschaft, Der internationale Vieh- und Fleischmarkt, Berlin, 1936-1944.

Reichsminister für Ernährung und Landwirtschaft, Reichsministerialblatt der landwirtschaftlichen Verwaltung, 1936-1945.

Id., Verordnungen zur Einführung der Kriegsernährungswirtschaft, 1939.

Reichsnährstand, Vieh- und Fleischwirtschaft, Ausgabe H, Berlin, 1935-1944.

\section{Bibliographie sélective}

Gustavo Corni, Horst Gies, Brot, Butter, Kanonen. Die Ernährungswirtschaft in Deutschland unter der Diktatur Hitlers, Berlin : Akademie Verlag, 1997.

John Edgar Farquharson, The Plough and the Swatiska. The NSDAP and Agriculture in Germany 1928-45, London : Sage Publications, 1976, p. 161-182.

Antje Frank, Das deutsche Dorf im zweiten Weltkrieg. Zu sozialen Erfahrungen und Verhaltensweisen von Bauern unterm Nationalsozialismus 1939 bis 1945, Uni. Rostock, Diss. für Philosophie, 1992.

Heinz Haushofer, Hans-Joachim Recke, 50 Jahre Reichsernährungsministerium Bundesernährungsministerium, Bonn: Bundesministerium für Ernährung, Landwirtschaft und Forsten, 1969.

Peter Haenger, Das Fleisch und die Metzger. Fleischkonsum und Metzgerhandwerk seit der Mitte des 19. Jahrhunderts, Zürich : Chronos, 2001. 
Ian Kershaw, L'Opinion allemande sous le nazisme, Bavière 1933-1945, Paris : CNRS Éditions, 1995, p. 59-84 et 260-269 sur l'opinion paysanne bavaroise et le IIIe Reich.

Richard Overy, War and Economy in the Third Reich, Oxford: Clarendon Press, 1994, p 259-314.

Henri Rouy, La Viande, Paris : PUF (Que sais-je ?, 374), 1949.

Hans Teuteberg, "Der Fleisch- und Wurstverzehr der Deutschen in historischer Betrachtung » in: Ernährungsforschung, 43/1, 1998, p. 1-28.

Ulrike Thoms, «Ernährung ist so wichtig wie Munition. Die Verpflegung der deutschen Wehrmacht 1933-1945 » in: Wolfgang U. Eckart, Alexander Neumann (dir.), Medizin im Zweiten Weltkrieg. Militärmedizinische Praxis und medizinische Wissenschaft im « Totalen Krieg» , Paderborn : Schöningh, 2006, p. 207-230.

Malte Zierenberg, Stadt der Schieber. Der Berliner Schwarzmarkt 1939-1950, Göttingen: Vandenhoeck \& Ruprecht, 2008

\section{NOTES}

1. Michel-Pierre Chélini, Inflation, État et opinion en France, 1944-1952, Paris : CHEFF, 1998, p. 421-437, 542-545.

2. Bertolt Brecht a également utilisé la métaphore de la viande abattue pour parodier le Horst Wessel Lied dans son drame Schweyk im Zweiten Weltkrieg (1943), avec une " Marche des veaux » (« Kälbermarsch »); B. Brecht, Gesammelte Gedichte, IV, Frankfurt a.M. : Suhrkamp, 1978, p. 1219-1220 : « Der Metzger ruft. Die Augen fest geschlossen / Das Kalb marschiert mit ruhig festem Tritt. / Die Kälber, deren Blut im Schlachthof schon geflossen / Sie ziehn im Geist in seinen Reihen mit.» («Le boucher appelle, les yeux complètement fermés. / Le veau défile d'un pas tranquille et ferme. / Ceux des veaux, dont le sang a déjà coulé dans l'abattoir, / s'insèrent en esprit dans les rangs. ») Le contenu du texte est parfois curieux en raison du souci de Brecht de parodier mot pour mot le premier couplet du Horst Wessel Lied. Ainsi du second vers, «SA marschiert mit ruhig festem Schritt.» («La SA défile d'un pas tranquille et ferme. ») Les vers 3 et 4 s'inspirent de l'évocation des âmes des SA abattus et qui accompagnent « en esprit » les SA vivants qui défilent.

3. ICREFH (Alain Drouard, dir.), Food and War in Europe in the Nineteenth and Twentieth Centuries, Paris, 8-11 septembre 2009.

4. Archives nationales, CARAN, Paris [désormais : AN], F/10/2172 : Viande - Marché de la viande et abattoirs, suivi 1902-1939. F/10/5107 : dossiers Adolphe Pointier, syndic de la Corporation nationale paysanne. F/10/5158-5172 : service des affaires économiques et des relations extérieures. Relations avec l'Allemagne. F/10/5208-5223 : service de statistiques et études agricoles. Production animale, viande. F/10/5623-26.

5. Bundesarchiv, Berlin Lichterfelde [désormais : BA] R 3601 : microfiches. La série BA R 3601 a regroupé deux anciennes séries BA R 14 et ZstA 3601, l'une du ministère de 
l'Agriculture du Reich, l'autre du ministère de l'Agriculture de Prusse, fusionnés en 1935-1938.

6. BA R $17 \mathrm{~V}$ : dossiers papiers.

7. Wilhelm Lenz, Reichsministerium für Ernährung und Landwirtschaft (Bestand R 14), Koblenz : Bundesarchiv (Findbücher zu Beständen des Bundesarchivs, 14), 1978. Heinz Boberach (éd.), Inventar archivalischer Quellen des NS-Staates, München: Saur, 1991-1995, Teil 1, p. 364-366, Teil 2, p. 246. Aujourd'hui Gorzów Wielkopolski, Landsberg est située à $45 \mathrm{~km}$ de Kostrzyn (Kustrin).

8. Nachlässe [« Papiers »] W. Darré et H. Backe.

9. BA R 16: Reichsnährstand. Reichshauptabteilung III. Bewirtschaftung einzelner Produkte, Marktberichte, usw. 1933-1945. BA R 16 I : Reichsbauernrat (aucune mention de la viande). BA R 68 II : Reichsverband der Rinderzüchter Deutschlands 1933-1936 (un dossier de $10 \mathrm{~cm}$ ).

10. La série BA R97 Reichsinnungsverband des Handwerkes porte seulement sur l'artisanat, la série BA R 12 III Reichsgruppe Handel n'incorpore pas la boucherie et la série BA R13 XXVI Wirtschaftsgruppe Lebensmittelindustrie. Fachgruppe Fleischwarenindustrie 1938, 1942 (1) ne compte qu'un dossier ! L'économie du Reich était organisée en sept Reichsgruppen, divisés en 54 Wirtschaftsgruppen, eux-mêmes articulés en un certain nombre de Fachgruppen. Une partie des dossiers centraux des sept Reichsgruppen ont disparu dans les flammes, tandis que sont conservées 34 Wirtschaftsgruppen sur 54, la part des Fachgruppen encore archivées étant elle-même très variable.

11. BA R 11: Reichswirtschaftskammer Nr. 2028-2029. Reichsinnungsverband des Fleischshandwerks (non consultés).

12. Définie selon un trapèze Islande-Finlande-Grèce-Portugal, sans l'URSS, l'Ukraine ou la Biélorussie, l'Europe couvre cinq millions de km2 pour 390 millions d'habitants en 1937.

13. UNO, Study about the European Food Situation in 1948, Genève, 1949, p. 18 suiv. INSEE, Études et conjoncture, économie mondiale, octobre-décembre 1947, p. 124-125 ; Henri Rouy, La Viande, Paris : PUF (Que sais-je ?, 374), 1949, p. 9 suiv.

14. Traditionnellement, depuis le XIXe siècle, les statistiques agricoles annuelles débutent le 1er juillet et se terminent le 30 juin. Les documents d'époque sont rarement en années civiles, lesquelles ont été reconstituées ensuite dans les années 1950-1960. Les moyennes quinquennales d'avant-guerre sont classiquement 1934-1938 en Europe comme pour les chiffres de la FAO en 1947.

15. Länderrat des Amerikanischen Besatzungsgebiets (éd.), Statistisches Handbuch für Deustchland 1928-44, München : Ehrenwirth, 1949, p. 232.

16. Statistisches Reichsamt, Statistisches Jahrbuch für das Deutsche Reich 1938, Berlin : Schmidt, p. 118-119 (chiffres disponibles depuis 1873).

17. H. Rouy, La Viande, op. cit., 1949, p. 8 suiv. Henri Rouy est ingénieur agronome spécialiste du marché de la viande dans les années 1930-1950. Son ouvrage présente des données extrêmement fiables pour l'époque. Pendant le conflit, il travaille avec le Comité [français] national interprofessionnel des viandes. Voir AN F/10/5158.

18. Sources: H. Rouy, La Viande, op. cit., p. 9. Länderrat des Amerikanischen Besatzungsgebiets (éd.), Statistisches Handbuch, op. cit., p. 231. Gustavo Corni, Horst Gies (dir.), Brot, Butter, Kanonen. Die Ernährungswirtschaft in Deutschland unter der Diktatur 
Hitlers, Berlin : Akademie Verlag, 1997, p. 487. UNO/FAO, Study about European Economic Situation and Perspectives in 1948, Genève, 1948, p. 12.

19. Länderrat des Amerikanischen Besatzungsgebiets (éd.), Statistisches Handbuch, op. cit., p. 231. G. Corni, H. Gies (dir.), Brot, op. cit., p. 487.

20. Statistisches Reichsamt, Statistisches Jahrbuch, op. cit., p. 118-119. Pour le détail du XIXe siècle, se reporter aux éditions antérieures, Kaiserliches Statistisches Amt, Statistisches Jahrbuch für das Deutsche Reich, Berlin : Puttkammer \& Mühlbrecht, 1re éd. 1879.

21. Länderrat des Amerikanischen Besatzungsgebiets (éd.), Statistisches Handbuch, op. cit., p. 232.

22. Statistisches Reichsamt, Statistisches Jahrbuch, op. cit., p. 118-119. À côté des 20,5 millions de bovins et des 23,8 millions de porcins recensés au 3 décembre 1937, le Reich compte 85,3 millions de poulets, 5,5 millions d'oies, 2,4 millions de canards.

23. En 1938 en Allemagne, $1 \mathrm{~kg}$ de bœuf à rôtir coûte 1,80 Reichsmark, $1 \mathrm{~kg}$ de bœuf à bouillir 1,69 RM, $1 \mathrm{~kg}$ de côtelette de porc 2,10 RM, $1 \mathrm{~kg}$ de viande de porc standard 1,68 $\mathrm{RM}, 1 \mathrm{~kg}$ de veau 2,23 RM, $1 \mathrm{~kg}$ de jambon 3,64 RM et $1 \mathrm{~kg}$ de lard/bacon 2,15 RM. Statistisches Bundesamt, Statistisches Jahrbuch für die BRD, Wiesbaden, 1952, p. 406.

24. Sondage réalisé en février 1946 en région parisienne. Institut français d'opinion publique, Sondages, Paris, 16 février 1946. Une enquête allemande équivalente n'a pas été trouvée.

25. «Carnaval » vient du latin « carnem levare », lever l'interdiction de viande.

26. L'incontournable rôti de porc allemand, Schweinebraten, avec pommes de terre et légumes.

27. H. Rouy, La Viande, op. cit., p. 15.

28. Länderrat des Amerikanischen Besatzungsgebiets (éd.), Statistisches Handbuch, op. cit., p. 214.

29. Statistisches Reichsamt, Statistisches Jahrbuch, op. cit., p. 118-119.

30. La concentration est peut-être un peu moins accentuée : les $1 \%$ les plus grandes ne rassemblent que $8 \%$ du cheptel, les $20-100$ ha qui forment $12 \%$ des exploitations capitalisent $28 \%$ du cheptel, tandis que les exploitations de moins de 5 ha représentent ici $47 \%$ de l'effectif exploitant et $21 \%$ des porcins. Pour les bovins, sur 100 exploitations, $41 \%$ disposent d'une à deux vaches, $46,5 \%$ de trois à six vaches et $12 \%$ de sept vaches et plus. Pour les porcins, $35 \%$ ont un ou deux porcs de plus de trois mois, $38 \%$ élèvent trois à dix porcs et $7 \%$ plus de onze porcs.

31. Statistisches Reichsamt, Statistisches Jahrbuch, op. cit., p. 122.

32. H. Rouy, La Viande, op. cit., p. 18.

33. Statistisches Reichsamt, Statistisches Jahrbuch, op. cit., p. 118.

34. Ibid., p. 125 et Länderrat des Amerikanischen Besatzungsgebiets (éd.), Statistisches Handbuch, op. cit., p. 215.

35. Statistisches Reichsamt, Statistisches Jahrbuch für das Deutsche Reich 1939-40, Berlin : Schmidt, 1942, p. 1.

36. H. Rouy, La Viande, op. cit., p. 10-11.

37. Les données pour 1941 n'ont pas été globalisées. Source: Länderrat des Amerikanischen Besatzungsgebiets (éd.), Statistisches Handbuch, op. cit., p. 231. 
38. Länderrat des Amerikanischen Besatzungsgebiets (éd.), Statistisches Handbuch, op. cit., p. 232.

39. Länderrat des Amerikanischen Besatzungsgebiets (éd.), Statistisches Handbuch, op. cit., p. 215. Le lait lui-même, dont la production baisse de $8 \%$, est totalement réquisitionné avec un pourcentage de $81 \%$ de la production à livrer. La part totale du lait frais recule de $31 \%$ en 1938 à $19 \%$ en 1944, tandis que la proportion de transformation en beurre, fromage, conserve, poudre augmente de 57 à $72 \%$. Le tonnage global de beurre produit augmente $(+23 \%)$, celui des fromages à pâte dure, de plus longue conservation, double pour compenser la crise de la viande, alors que les fromages à pâte tendre restent stables et que le fromage blanc frais (Speisequark) recule.

40. Katharina Hoffmann, « Zwangsarbeit in der Landwirtschaft » in : Ulrike Winkler (dir.), Stiften gehen. NS-Zwangsarbeit und Entschädigungsdebatte, Köln : PapyRossa, 2000, p. 130-147 ; Ead., « Zwangsarbeiter in der deutschen Landwirtschaft 1939-1945 » in : Ulrich Herbert (dir.), Europa und der «Reichseinsatz». Ausländische Zivilarbeiter, Kriegsgefangene und KZ-Häftlinge in Deutschland 1938-1945, Essen : Klartext, 1991, p. 127-139.

41. Immortalisé en mode comique par Fernandel dans La Vache et le prisonnier, dir. Henri Verneuil, 1959, $35 \mathrm{~mm}$, noir et blanc, $1 \mathrm{~h} 59$.

42. Statistisches Bundesamt, Statistisches Jahrbuch für die BRD, op. cit., p. 452, 464.

43. Ibid., p. 467. Moyenne de 72 communes allemandes. $1 \mathrm{~kg}$ de viande de bœuf à bouillir avec os coûte 1,67 RM, $1 \mathrm{~kg}$ de viande de porc (poitrine) 1,63 RM, $1 \mathrm{~kg}$ de veau (poitrine) 2,06 RM, $1 \mathrm{~kg}$ de lard 2,14 RM, $1 \mathrm{~L}$ de lait entier (en 1944) 0,25 RM, $1 \mathrm{~kg}$ de beurre de laiterie (en 1944) 3,59 RM.

44. Ibid., p. 468-472. Revenu brut hebdomadaire des ouvriers en moyenne annuelle. Certaines catégories sont plus encouragées comme les mineurs de charbon dont le revenu nominal progresse de $20 \%$.

45. BA R 3601/3371 : Versorgungslage betreffend Getreide und Fleisch 1941. Plusieurs rapports et courriers, dont celui de Goering en date du 13 janvier 1941.

46. BA R 3601/3154a: Därme, 23. Januar 1941, RMEL. Abgrenzung des Zuständigkeitsbereichs zwischen REM und dem Reichswirtschaftsministerium, Schreiben vom 17. Dezember 1940.

47. BA R 17 V 29 : Viehzählung 1936-1943.

48. BA R 3601/3162 : Selbstversorgung mit Fleisch und Fett 1942-1944. BA R 3601/3179 : Selbstversorgung mit Fleisch und Fett, Hausschlachtungen. BA R 17 V 14 : Fleischbilanzen 1942-1945. Un film récent, Marga, qui se joue en Westphalie en 1943-1945, fait droit à la scène d'abattage clandestin d'un porc, dans laquelle les fermiers impliquent le responsable local des Jeunesses hitlériennes pour obtenir son silence (titre original: Unter Bauern, dir. Ludi Boeken, 2009, couleur, 1h40).

49. BA R 3601/3179. Au Nord, à l'Ouest et à l'Est, on compte $175 \mathrm{~kg}$ de poids vif pour 140 $\mathrm{kg}$ de viande nette, soit la consommation maximale pour trois personnes et demie, au Sud et au Sud-Est, $150 \mathrm{~kg}$ vifs, soit $120 \mathrm{~kg}$ nets annuels pour trois personnes.

50. BA R 17 V 27 : Märkte, Mittelmärkte, Verteilungsstellen. Commissions de classification des unions d'éleveurs de bétail (Klassifizierungs-Kommissionen der Viehwirtschaftsverbände). 
51. BA R 3601/3154: Fleischversorgung des Protektorates Böhmen und Mähren. Fleischbilanz im Böhmen-Mähren, 1. September 1940-31. August 1941.

52. BA R 17 V 27 : Märkte, Mittelmärkte, Verteilungsstellen. Notamment la liste des abattoirs avec connexion ferroviaire au 1er juillet 1943 (Schlachthöfe bzw. Schlacht- und Viehhöfe mit Gleisanschluss, Stand 1. Juli 1943).

53. BA R 17 V 27 : Märkte, Mittelmärkte, Verteilungsstellen. Stettin peut servir d'exemple avec une capacité de 1000-1 200 bovins par semaine. Les jours d'abattage sont le lundi et le mercredi, et donc les jours de marché mardi et jeudi. Le marché communal (Marktgemeinschaft) regroupe l'agglomération de Stettin et quelques localités contiguës.

54. BA R 17 V 14 : Fleischbilanzen 1942-1945. « 46. Woche vom 15.11-22.11.1943 vorläufig. 49 Großmärkte ohne Niedersachsen, Kurhessen und Husum. Auftriebe. Summe : 18356 Rinder, 32241 Kälber, 15340 Schafe, 81589 Schweine usw. » («Bilans de la production de viande, 1942-1945. 46e semaine du 15 au 22 novembre 1943, résultats provisoires. 49 marchés de gros, excepté Basse-Saxe, Hesse-Cassel et Husum [dans le Schleswig-Holstein]. Activité. Total : 18356 bœufs, 32241 veaux, 15340 moutons, 81589 porcs, etc. »)

55. BA R 17 V 115: Schlachtviehmarktordnung 1943. Genossenschaftliche ReichViehverwertung mbH, Anordnung Nr. 1 der HDV betreffend Schlachtviehmarktordnung für das Jahr 1943, 18. Dezember 1942.

56. BA R 3601/3154 : Fleischversorgung des Protektorates Böhmen und Mähren.

57. BA R 17 V 27 : Märkte, Mittelmärkte, Verteilungsstellen.

58. BA R 3601/3154a: Arbeitsbericht der Reichsfachschaft der Darm- und Innereienkaufleute im Reichsnährstand und der Fachgruppe Fleischereibedarf in der Wirtschaftsgruppe Groß-, Ein- und Ausfuhrhandel 1933-1941.

59. BA R 3101 : Reichswirtschaftsministerium, R 11 Reichswirtschaftskammer Nr. 2028-2029, Reichsinnungsverband des Fleischshandwerks (dossiers non consultés).

60. G. Corni, H. Gies (dir.), Brot, op. cit., p. 487-489.

61. Ibid., p. 555-582.

62. Ulrike Thoms, «Ernährung ist so wichtig wie Munition. Die Verpflegung der deutschen Wehrmacht 1933-1945 » in : Wolfgang U. Eckart, Alexander Neumann (dir.), Medizin im Zweiten Weltkrieg. Militärmedizinische Praxis und medizinische Wissenschaft im « Totalen Krieg », Paderborn : Schöningh, 2006, p. 207-230.

63. BA R 3601/3153 : Fleischversorgung der Wehrmacht 1943-1944.

64. U. Thoms, « Ernährung ... », op. cit., p. 221 ; calculs à partir de BA N 1198/49 et BA-MA [Freiburg] RH 12-23/1640 et 1643.

65. BA R 3601/3153 : Fleischversorgung der Wehrmacht 1943-1944.

66. Avec des chiffres parfois difficiles à interpréter : les besoins de la Kriegsmarine en 1944 sont évalués à 29000 t. par son haut commandement, ce qui correspondrait à près de $1 \mathrm{~kg}$ de viande par marin (environ 80000 marins) et par jour ! Peut-être cela inclut-il les tonnages dont la Kriegsmarine assure le ravitaillement par mer. BA R 3601/3153 : Fleischversorgung der Wehrmacht 1943-1944. Oberkommando der Kriegsmarine, 28. Januar 1944. Übermittlung am 16. Februar 1944 vom RMEL an die Hauptvereinigung der Deutschen Viehwirtschaft.

67. Pratique signalée en plusieurs endroits. AN, F/10/5158: relations avec le commandement militaire, hôtel Majestic, 1941-1944, entretien du 10 mars 1942. En 
1942-1943, la Luftwaffe emploie directement plus de deux millions de personnes : pilotes, FLAK antiaérienne, radio et renseignement, terrassement et génie aérien, police et protection, sans compter les auxiliaires et les civils en renfort qui portent le total à près de trois millions.

68. BA R 17 V 14 : Fleischbilanzen 1942-1945.

69. BA R 3601/3153 : il fournissait $10 \mathrm{t}$. par semaine au début du conflit, 5,5 t. en automne 1943 avec 70 animaux, et demande une extension [refusée] de son abattoir. Brief am 23. September 1943 vom Regierungspräsident des Regierungsbezirks Potsdam an den Oberregierungsrat Graf von Brühl im RMEL.

70. BA R $3601 / 3153$ : 22. Februar 1944, geheim. Diskussion zwischen Wehrmacht und RMEL über die Anrechnung von Eiern und Trockeneipulver in Kalorien und in Fleischkapazität.

71. BA R 3601/3153 : Fleischversorgung der Wehrmacht 1943-1944, Dokument 121, 17. Oktober 1944.

72. Wurstkonserven: boudin (Blutwurst), saucisse de foie (Leberwurst), saucisse " chasseur " (Jagdwurst) à base de bœuf et de porc incomplètement hachés et saucisse pour accompagner la bière (Bierwurst bavaroise), faite de morceaux de bœuf, de porc et de cœur. BA R 3601/3153 : Fleischversorgung der Wehrmacht 1943-1944. Dokument 121, 17. Oktober 1944.

73. BA R 3601/3153 : Fleischversorgung der Wehrmacht 1943-1944. Office du Reich pour les Produits animaux, lettre du 12 août 1944 au ministère du Ravitaillement.

74. BA R 3601/3153 : Fleischversorgung der Wehrmacht 1943-1944. Lettre du 6 octobre 1944 de l'OKH et rapport du 17 octobre 1944 de l'office du Reich pour les Produits animaux.

75. BA R 3601/3154b : Fleischkonserven - Herstellung, Verteilung, Preisbildung 1939-1944.

76. Ernst Piecszek, « Neuzeitliche Gedanken auf dem Gebiet der Verpflegung » in : Ernst Piesczek, Wilhelm Ziegelmayer, Ernährung der Wehrmacht, 1. Tagungsbericht der Arbeitsgemeinschaft Ernährung der Wehrmacht, Dresden/ Leipzig: Th. Steinkopff, 1942, p. 15 ; cité par U. Thoms, « Ernährung ... », op. cit., p. 227.

77. BA R 3601/3189 : Bau von Tiefkühlanlagen. Construction de réfrigérateurs de grande capacité pour la viande, les légumes et autres aliments. Le gros de la correspondance archivée date de 1943-1944 et concerne les nouveaux procédés, la mise en place de matériels de réfrigération, mais le dossier est maigre (une centaine de pages en microfiches). Une demi-douzaine d'entreprises est en relation avec le ministère du Ravitaillement.

78. U. Thoms, « The Innovative Power of War. Army, Food Sciences and the Food Industry in Germany in the 20th Century ", Food and War in Europe in the Nineteenth and Twentieth Centuries, Paris, 8-11 septembre 2009.

79. BA R $3601 / 3154 \mathrm{~d}$ : Fleischbewirtschaftung.

80. BA R $3601 / 3154 d$.

81. Commission consultative des dommages et réparations, Dommages subis par la France et l'Union française, du fait de la guerre et de l'occupation ennemie, 1939-1945. Part imputable à l'Allemagne, Paris, Imprimerie nationale, 1950, I, p. XIX : 890000 t. en quatre ans, soit $16 \%$ de l'offre moyenne annuelle de viande en France. 
82. BA R 17 V 14 : Fleischbilanzen 1942-1945. Synthèse de rapports et de correspondances sur les livraisons de la France, septembre 1942-août 1943.

83. AN, F/10/5158 : relations avec le commandement militaire, hôtel Majestic, 1941-1944. Secrétariat d'État à l'Agriculture, service des affaires économiques et des relations extérieures. Le dossier contient les rapports, la correspondance et les procès-verbaux des échanges avec le MBHF pour l'agriculture et le ravitaillement, dans lesquels la viande ou le bétail peuvent apparaître. Par exemple, dans l'entretien du 27 juillet 1942, le point 1 concerne les «mesures techniques d'exécution concernant la livraison des 16000 tonnes de viande pour le mois d'août », sans autre précision.

84. BA R 3601/3153: Fleischversorgung der Wehrmacht 1943-1944. Rapport du 23 décembre 1943, faisant étant d'un entretien entre le directeur du ministère du Ravitaillement et l'OKH.

85. BA R 3601/3153 : Fleischversorgung der Wehrmacht 1943-1944.

86. BA R 17 V 14 : Fleischbilanzen 1942-1945. Bevollmächtigter General der deutschen Wehrmacht in Italien, Verwaltungsstab. Riva an die HDV. Lettre du 11 novembre 1943.

87. BA R 17 V 14 : Fleischbilanzen 1942-1945. Télégramme du RMEL au Reichsleiter Martin Bormann, 10 novembre 1944, opération évacuation du bétail de Prusse orientale (Räumungsviehaktion aus Ostpreußen), qui a concerné 132000 bœufs, 19400 porcs et 56 000 moutons, évacués le 25 octobre 1944 à l'intérieur du Reich.

88. BA R 3601/3153 : Rundbrief des RMEL an alle Landesernährungsämter, Abt. A und B, 2. September 1944.

89. Voir Werner Abelshauser, Wirtschaft in Westdeutschland 1945-48, Rekonstruktion und Wachstumsbedingungen in der Amerikanischen und Britischen Zone, Stuttgart: Deutsche Verlags-Anstalt, 1975, p. 132-138.

90. Statistisches Bundesamt, Statistisches Jahrbuch für die BRD, op. cit., p. 400-404 pour les prix alimentaires, p. 144 pour l'état du cheptel. Statistisches Amt der DDR, Statistisches Jahrbuch der DDR, Berlin: Haufe, 1960, p. 479 suiv.; les données commencent en 1950 quand l'état du bétail atteint approximativement les niveaux de 1938.

91. Statistisches Bundesamt, Vom Erzeuger zum Verbraucher: Fleischversorgung in Deutschland. Ausgabe 2008, Wiesbaden : Stat. Bundesamt, 2008. 